\title{
Clinicopathological examination of ESD as salvage therapy for esophageal cancer after definitive chemo-radiation therapy
}

\section{(c) $(>)$}

\author{
Authors \\ Mizobuchi ${ }^{3}$ \\ Institutions \\ 1 Department of Gastroenterology, Kagawa Prefectural \\ Central Hospital, Takamatsu, Japan \\ 2 Department of Medical Oncology, Kagawa Prefectural \\ Central Hospital, Takamatsu, Japan \\ 3 Department of Pathology, Kagawa Prefectural Central \\ Hospital, Takamatsu, Japan
}

Tomo Kagawa', Shigenao Ishikawa', Tomoki Inaba', Mariko Colvin', Junki Toyosawa', Yuki Aoyama', Masaya Ishida', Sakiko Kuraoka ${ }^{1}$, Kunio Okamoto ${ }^{1,2}$, Ichiro Sakakihara ${ }^{1}$, Koichi Izumikawa ${ }^{1}, K_{\text {Kumo Yamamoto }}{ }^{1}$, Sakuma Takahashi ${ }^{1}$, Shigetomi Tanaka1, Mihoko Matsuura', Toshimi Hasui ${ }^{1}$, Masaki Wato', Midori Ando ${ }^{3}$, Satoko Nakamura ${ }^{3}$, Koichi

submitted 29.7.2017

accepted after revision 8.1.2018

\author{
Bibliography \\ DOI https://doi.org/10.1055/s-0044-102296 | \\ Endoscopy International Open 2018; 06: E450-E461 \\ (c) Georg Thieme Verlag KG Stuttgart · New York \\ ISSN 2364-3722
}

Corresponding author

Shigenao Ishikawa, MD, Department of Gastroenterology, Kagawa Prefectural Central Hospital, 1-2-1 Asahi-machi, Takamatsu, 760-8557, Japan

Fax: +81-87-802-1188

ishikawa5308@yahoo.co.jp

\section{ABSTRACT}

Background and study aims Salvage therapy for esophageal cancer following chemo-radiation therapy (CRT) has not been established. We aimed to evaluate endoscopic submucosal dissection (ESD) as a salvage therapy based on histopathological features of lesions.

Patients and methods We compared 10 lesions in eight patients with local residual, recurrent, or metachronous esophageal squamous cell carcinoma treated by ESD after CRT (CRT group) and 59 lesions treated by ESD without CRT (non-CRT group) during the same period.

Results The en bloc resection rate was $100 \%$ while the complete resection rate was $80.0 \%$ in the lesions after $C R T$, indicating no difference between the CRT and nonCRT groups. Pathological examination showed that fibrosis was more intense in the lamina propria mucosa, muscularis mucosa, and submucosa. The muscularis mucosa was thicker in both non-tumor and tumor sites in the CRT group compared to the non-CRT group. However, severe submucosal fibrosis was observed only in one lesion in the CRT group. The maximum diameter of the submucosal artery was significantly larger in the CRT group $(P<0.001)$.

Conclusions Compared to the non-CRT group, the lesions in the CRT group were accompanied by fibrosis while the muscularis mucosa were thicker; however, severe fibrosis of the submucosa was rare. It is important to dissect the muscularis mucosa appropriately during ESD, which makes successful dissection of the submucosa possible. Attention should be paid to bleeding from large arteries.

\section{Introduction}

Treatment outcomes with chemo-radiation therapy (CRT) for esophageal cancer are reportedly comparable to those with surgery. Additional surgical resection has been widely used as salvage therapy for esophageal squamous cell carcinoma after CRT. However, its application is limited due to complications and perioperative mortality as high as $7 \%$ to $8 \%[1,2]$. Although the application of CRT is expanding, the rate of local residual disease or recurrence after CRT is consistently high, ranging from $25 \%$ to $40 \%$ [3-6]. In addition, patients with esophageal squamous cell carcinoma (SCC) are at risk for development of multiple SCCs $[7,8]$ and sometimes develop a second primary SCC within the RT field. The rate of metachronous cancer after CRT has been reported as $22 \%$ [9]; that is high enough to be an important issue. Thus, due to unexpected outcomes, contro- 
versy exists regarding salvage therapy. There are few reports on endoscopic submucosal dissection (ESD) [10-12] despite many reports describing endoscopic mucosal resection (EMR) [13-15]. Locoregional failure or a metachronous lesion after CRT may be accompanied by tissue fibrosis $[10,11,16]$, which may cause difficulty in performing ESD and may lead to accidental perforation or massive bleeding during the procedure.

In this study, we retrospectively examined the histopathology of esophageal lesions with SCC obtained by ESD after definitive CRT with the aim of clarifying the relationship between histopathology and adequacy of the procedure.

\section{Patients and methods}

From eight patients with esophageal SCC, 10 specimens were obtained by ESD as salvage therapy after definitive CRT (CRT group) in our hospital from April 2010 to December 2016. These specimens were histopathologically compared with 59 lesions in 54 consecutive patients with esophageal SCC in whom CRT was not performed (non-CRT group) during the same period. Based on the Japanese Esophageal Society Guideline [17], site of occurrence, macroscopic type, and histopathological findings of the lesions were examined. In each patient, ESD was performed at the site of residual or recurrent lesions after CRT.

Lesions of esophageal cancer were classified as follows.

Residual tumor: cancer confirmed immediately after CRT endoscopically or histopathologically.

Recurrent tumor: recurrence after having once achieved a complete response after CRT, in the area where the primary tumor had existed prior to CRT. Biopsy findings were positive for cancer cells from recurrent lesions.

Metachronous lesion: secondary cancer detected in different site from the primary lesion after CRT by endoscopy.

Two expert endoscopists with experience in $>200$ cases of ESD for gastric tumors performed all ESDs in same manner in both groups. A dual knife (KD-650Q, Olympus Medical Systems, Co., Tokyo, Japan) and high-frequency generator (VIO300D, VIO 300 D, ERBE, Tübingen, Germany) were used for ESD. After marking around the lesions using soft coagulation (effect 2, 20 W), sodium hyaluronate $0.25 \%$ mixed with glyceol or sodium hyaluronate $0.4 \%$ was locally injected into the submucosa followed by mucosal resection using Endocut I (effect 1, duration 2, interval 3). Swift coagulation (effect 2, 20W) was used for submucosal dissection. A small-caliber-tip transparent hood (DH-28GR; Fujifilm Medical System Co, Ltd., Tokyo, Japan) was attached to the end of the endoscope, if necessary.

Pathological examination of tumor lesion was performed to verify that the tumor was completely excised including $2 \mathrm{~mm}$ of a normal margin site (non-tumor site). The resected specimens were cut into 2 -mm slices, fixed with formalin solution, and were embedded in paraffin solution and further cut into $3-\mu \mathrm{m}$ thick sections for hematoxylin-Eosin and azan staining. At tumor and non-tumor sites, the thickness of the lamina propria mucosa and the muscularis mucosa was measured. The value of the thickness of the lesions was defined as an average of the maximum and the minimum thickness.

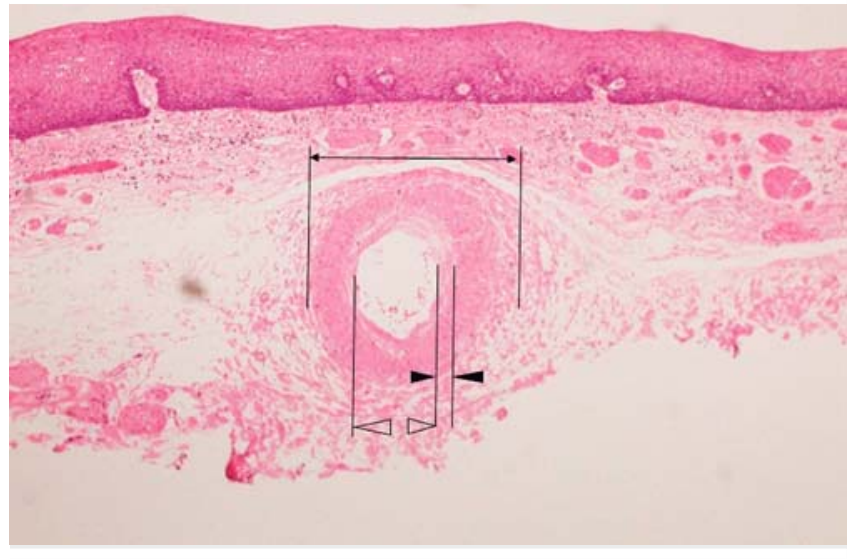

- Fig. 1 Submucosal artery. The outer diameter (arrow), diameter of lumen (white arrowhead) of the largest blood vessel, where the long diameter and the short diameter are the same, was measured. The black arrowhead indicates the vascular endothelial thickness.

The vessel diameter of the submucosal artery and vein of the stained light microscopic images at $\times 200$ magnification was measured. The diameter was defined as the outer diameter and the arterial lumen of the largest vessel, where the long and short diameters were the same considering the effect of the cutting angle of the pathological section ( $\triangleright$ Fig. 1 ). Histopathological measurements were independently performed by two specialists, and the mean value was used for this analysis.

The degree of fibrosis of the lamina propria mucosae, the cularis mucosa, and the submucosa was defined as mild, moderate, or severe, according to the criteria described below ( $\vee$ Fig. 2, $>$ Fig. 3, $>$ Fig.4). Based on observation of five fields at medium magnification $(\times 200)$ and 10 fields at high power $(\times 400)$, fibrosis was defined as mild (when fibrous tissue was less than $30 \%$ ), moderate (fibrous tissue was between $30 \%$ and $60 \%$ ), or severe (fibrous tissue was $60 \%$ or more.) Azan staining was used for supplementary evaluation of fibrosis ( $\triangleright$ Fig.5). Because moderate fibrosis induced by radiation was the target (for evaluation) in this study, histopathological assessment was performed by the specialist at both tumor and non-tumor sites. Details of the clinical information were masked to observers. Semiquantitative histopathological analysis was thought to be sufficient for disregarding influences from tumor-induced fibrosis or clinical usage of ESD. Because a portion of a tumor may become fibrous because of either fibrosis influenced by the tumor itself or biopsy, we divided the lesion into tumor and non-tumor sites for pathological examination measurements and comparison of the lesions. A healthy margin of $2 \mathrm{~mm}$ on average was resected around the tumor and examined as part of the non-tumor site.

IBM SPSS Statistics Ver.22 was used for statistical processing. Chi-square test was used for the qualitative variable test while Mann-Whitney's $U$ test was used for the quantitative variable test. The difference was considered significant when $P<0.05$.

The study was conducted in accordance with the Declaration of Helsinki and under the approval the Ethics Committee. Written informed consent was obtained from all patients. 

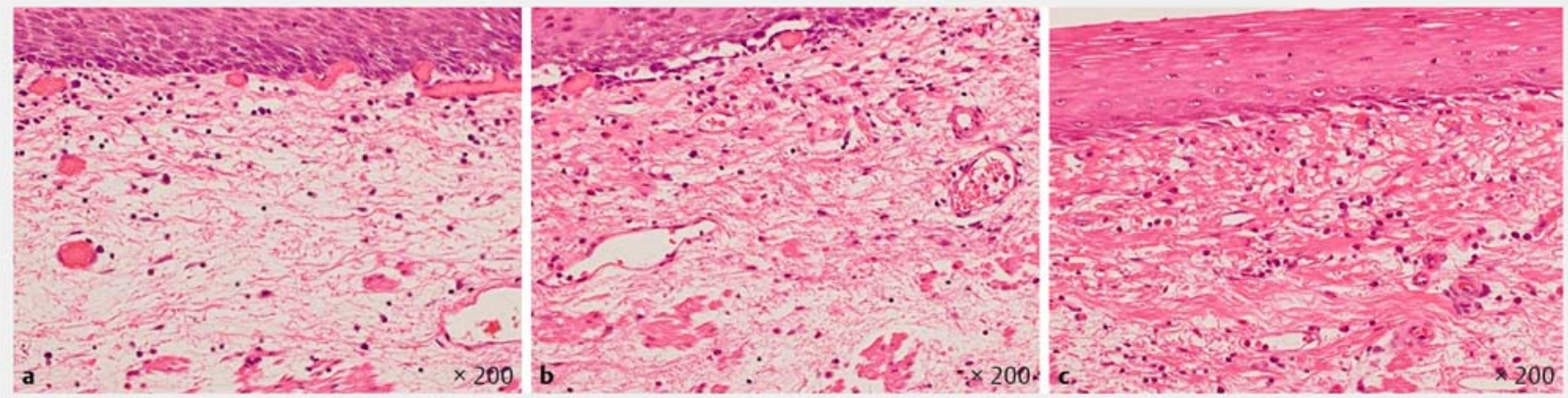

- Fig. 2 Fibrosis of the lamina propria mucosa in the CRT group. a Mild fibrosis: less than $30 \%$ fibrosis is observed (200×magnification). b Moderate fibrosis: $30 \%$ to $60 \%$ fibrosis is observed ( $200 \times$ magnification). c Severe fibrosis: $60 \%$ or more fibrosis is observed ( $200 \times$ magnification).
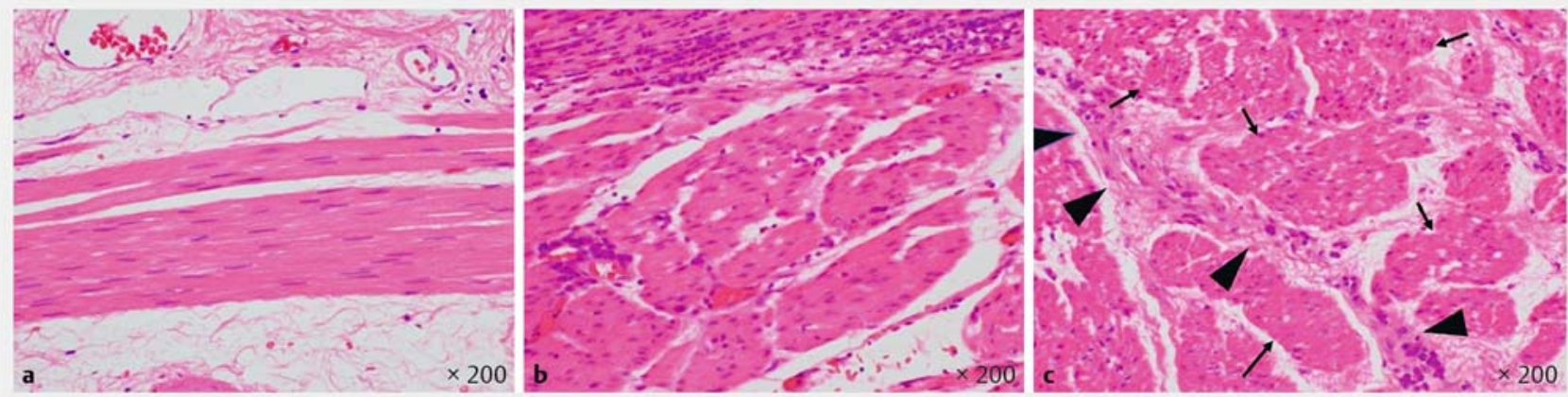

- Fig. 3 Fibrosis of the muscularis mucosa in the CRT group. a Mild fibrosis. Fewer than $30 \%$ collagen fibers (arrowhead) contacted by fibroblasts between the smooth muscle cells (arrow), with a nucleus at the center of the cell. b Moderate fibrosis. c Severe fibrosis.
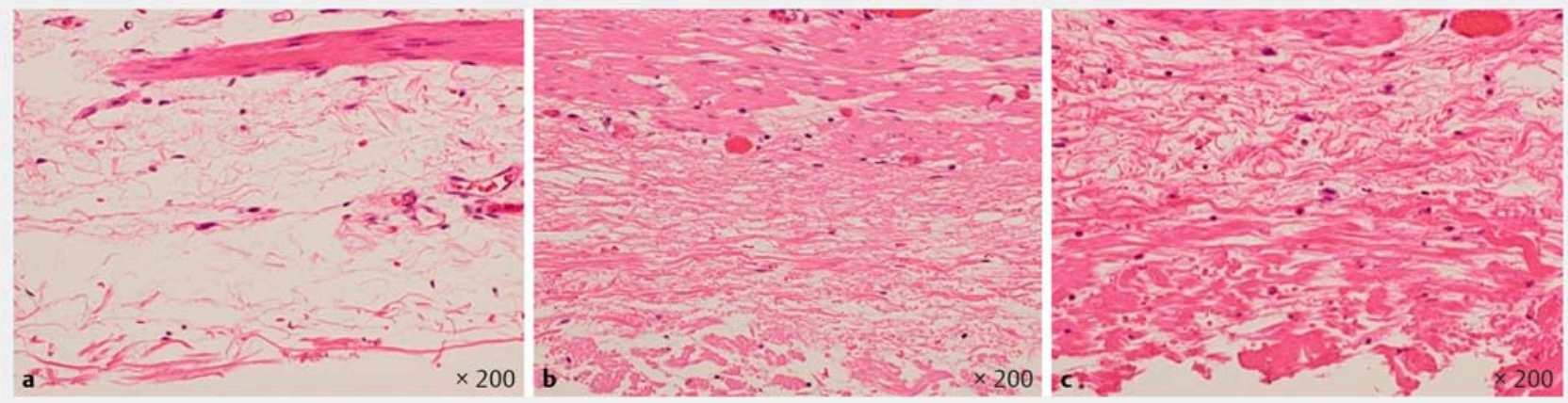

Fig. 4 Fibrosis of the submucosa. a Mild fibrosis. b Moderate fibrosis. c Severe fibrosis.

\section{Results}

\section{Background of patients in the CRT group}

Lesions consisted of four metachronous, three residual, and three recurrent lesions; the metachronous lesions arose within the original RT field. Two local failure lesions were detected approximately 8 years after CRT (lesions 3 and 6). Previous study [9] reported that recurrence exceeding 5 years after CRT is very rare. These lesions were therefore thought unlikely to be recur- rent and were classified as metachronous lesions. The site of lesions was the cervical esophagus (Ce) in two lesions, the middle thoracic esophagus (Mt) in five lesions, the lower thoracic esophagus (Lt) in two lesions, and the abdominal esophagus (Ae) in one lesion. The stage of the carcinoma prior to CRT was I in six patients, II in one, and III in one. For CRT, 5-fluorouracil and cisplatin were administered in combination with radiation at 60 Gy ( $>$ Table 1$)$. 

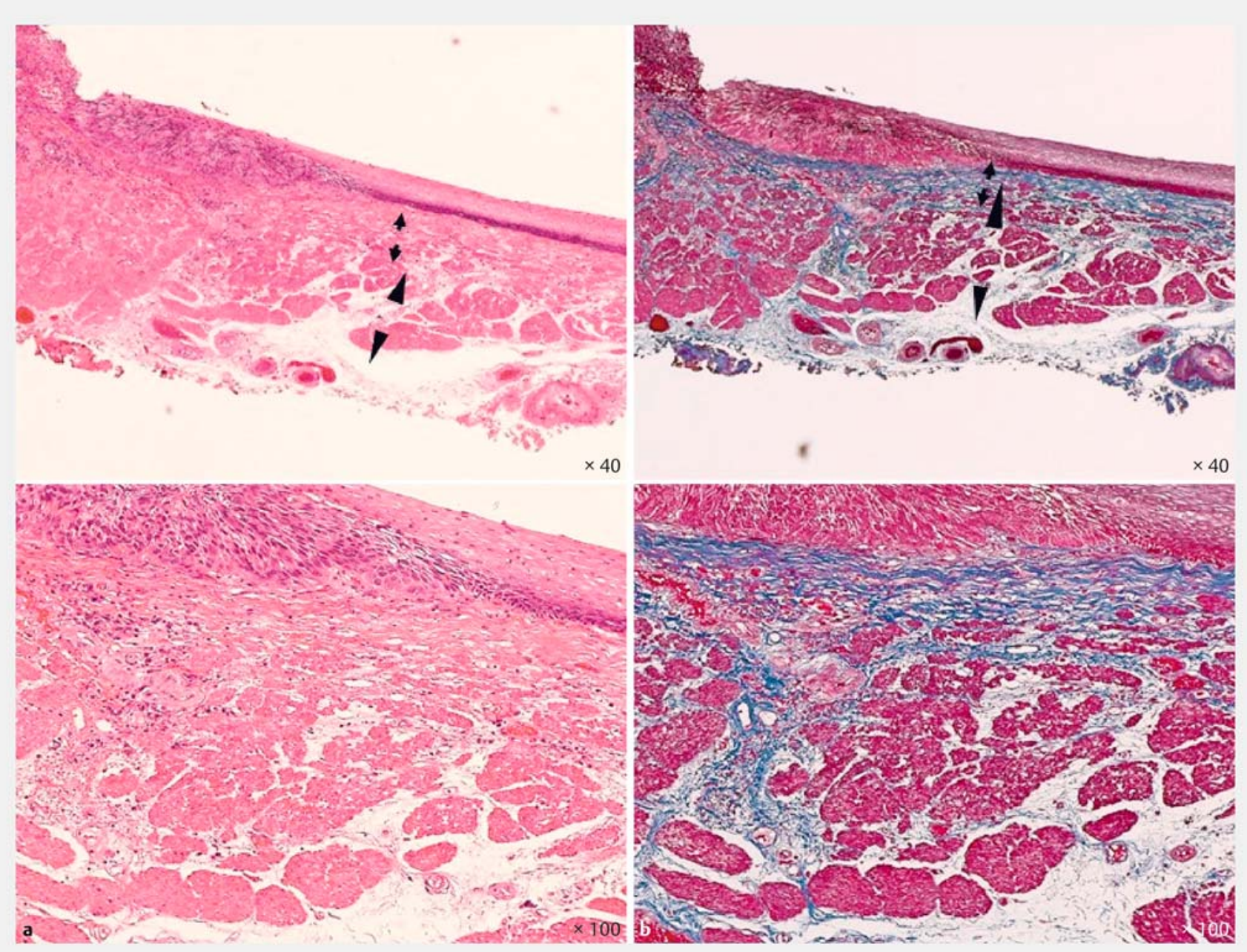

- Fig. 5 Azan staining of the muscularis mucosae in the CRT group. Collagen fibers are stained clearly in dark blue. The arrow indicates the lamina propria mucosa, while the arrowhead indicates muscularis mucosa. a Hematoxylin-Eosin stain. b Azan stain.

There were no accidental events such as bleeding, perforation, or postoperative stenosis associated with ESD. The invasion depth was T1a-EP in five lesions, T1a-LPM in three, and T1a-MM in two. The lateral margin could not be evaluated in two lesions due to damage to the lesions during dissection. Invasive type was INF $\alpha$ in all lesions and no vascular invasion was observed ( $\triangleright$ Table 2). Endoscopic examination was conducted every 6 months and follow-up via computed tomography without additional treatment after ESD demonstrated that there was no recurrence in any patient, with an average follow-up period of 11.9 months.

\section{Comparison of clinical features of the CRT group and the non-CRT group}

No statistically significant difference was observed in sex, age, lesion sites, invasion depth, or size of resected section between groups ( $>$ Table 3 ). Median duration of ESD was 42.0 minutes in the CRT group and 56.5 minutes in the non-CRT group. No significant difference was observed in treatment outcome (en bloc resection rate, complete resection rate, and curative resection rate) between groups.

\section{Comparison of histopathological findings between the CRT group and non-CRT group}

The thickness of the lamina propria mucosa in both non-tumor and tumor sites in the CRT group was significantly greater than that in the non-CRT group (overall $P<0.001$ ) ( $>$ Table 4). Degree of fibrosis in the lamina propria mucosa in both non-tumor and tumor sites in the CRT group was significantly higher than that in the non-CRT group $(P=0.002$ and $P=0.003$, respectively). The thickness of the muscularis mucosae in both non-tumor and tumor sites in the CRT group was significantly greater than that in the non-CRT group (overall $P<0.001$ ). Fibrosis was observed within the muscularis mucosa of all patients in the CRT group and the degree of fibrosis was significantly higher than that in the non-CRT group in both non-tumor and tumor sites (overall $P<0.001$ ). In the CRT group, the degree of fibrosis of the submucosa was significantly higher than that in the non- 
- Table 1 Primary tumors in the CRT group.

\begin{tabular}{|c|c|c|c|c|c|c|c|c|c|}
\hline Case & Age & Sex & $\begin{array}{l}\text { Site of } \\
\text { primary } \\
\text { tumor }\end{array}$ & $\begin{array}{l}\text { Clinical } \\
\text { T }\end{array}$ & $\begin{array}{l}\text { Clinical } \\
\mathrm{N}\end{array}$ & $\begin{array}{l}\text { Clinical } \\
\text { M }\end{array}$ & $\begin{array}{l}\text { Clinical } \\
\text { stage }\end{array}$ & Problems in surgery & $\begin{array}{l}\text { Treatment } \\
\text { of primary } \\
\text { tumor }\end{array}$ \\
\hline 1 & 68 & M & Mt & $\mathrm{T} 2$ & N2 & M0 & Stage III & $\begin{array}{l}\text { Angina pectoris (post-CABG } \\
\text { surgery) }\end{array}$ & FP-RT ${ }^{1}$ \\
\hline 2 & 48 & M & Ut Mt & $\mathrm{T} 1 \mathrm{~b}$ & N2 & Mo & Stage II & $\begin{array}{l}\text { Atrial fibrillation, chronic heart } \\
\text { failure, post-distal gastrectomy } \\
\text { for advanced gastric cancer }\end{array}$ & FP-RT ${ }^{1}, \mathrm{DCF}^{2}$ \\
\hline 3 & 52 & M & $\mathrm{Ce}$ & $\mathrm{T} 1 \mathrm{~b}$ & No & Mo & Stage I & $\begin{array}{l}\text { Due to the lesion was in Ce, total } \\
\text { laryngectomy is required in the } \\
\text { case of surgery }\end{array}$ & FP-RT ${ }^{1}$ \\
\hline 4 & 79 & $\mathrm{M}$ & $\mathrm{Mt}$ & $\mathrm{T} 1 \mathrm{~b}$ & No & M0 & Stage I & Old age & $\mathrm{FP}^{-R T^{1}}$ \\
\hline 5 & 63 & M & Ut Mt & $\mathrm{T} 1 \mathrm{~b}$ & NO & Mo & Stage I & $\begin{array}{l}\text { Old myocardial infarction, empye- } \\
\text { ma pleurae }\end{array}$ & FP-RT ${ }^{1}$ \\
\hline 6 & 56 & $\mathrm{~F}$ & Mt & $\mathrm{T} 1 \mathrm{~b}$ & No & Mo & Stage I & $\begin{array}{l}\text { Undetermined connective } \\
\text { tissue disease }\end{array}$ & ESD, $F P-R T^{1}$ \\
\hline 7 & 66 & M & Mt & $\mathrm{T} 1 \mathrm{~b}$ & No & M0 & Stage I & $\begin{array}{l}\text { Post-mitral valve replacement } \\
\text { surgery (mechanical valve), } \\
\text { atrial fibrillation, COPD, cerebral } \\
\text { infraction sequela }\end{array}$ & FP-RT ${ }^{1}$ \\
\hline 8 & 80 & M & Mt & $\mathrm{T} 1 \mathrm{~b}$ & No & M0 & Stage I & Angina pectoris, diabetes, old age & FP-RT ${ }^{1}$ \\
\hline
\end{tabular}

CRT group, in both non-tumor and tumor sites (overall $P<$ $0.001)$.

In the muscularis mucosa of the CRT group, mild fibrosis was observed in 1 of 10 patients (10\%), moderate fibrosis in 7 of 10 (70.0\%) and severe fibrosis in 2 of $10(20.0 \%)$ in non-tumor sites; mild fibrosis was present in 1 of $10(10.0 \%)$, moderate fibrosis in 6 of $10(60.0 \%)$, and severe fibrosis in 3 of $10(30.0 \%)$ in the tumor site. On the other hand, in the submucosa of the CRT group, mild fibrosis was observed in 3 of 10 patients (30.0\%) and moderate fibrosis in 7 of 10 (70.0\%) in non-tumor sites; no severe fibrosis was observed in the non-tumor sites; mild fibrosis was observed in 3 of 10 patients (30.0\%), moderate fibrosis in 6 of 10 (60.0\%), and severe fibrosis in only 1 of 10 $(10.0 \%)$ in the tumor site ( $\triangleright$ Table 5 ).

There was no difference in the maximum diameter of the submucosal vein while the artery and artery lumen diameters in the CRT group were significantly larger than those in the non-CRT group $(P<0.001, P<0.001$, respectively) ( $\vee$ Table 4$)$.

\section{Discussion}

Treatment for esophageal cancer after CRT has become the major concern recently. Yano et al. [14] used EMR, a less invasive treatment, and concluded that the en bloc resection rate was still as high as $33 \%$ while the local recurrence rate after EMR was also high at $28.6 \%$. Makazu et al. [18] reported many cases related to EMR after CRT. They performed EMR treatment in 11 cases of residual or recurrent lesions after CRT, with 13 lesions total. The major difference from our examination lies in the en bloc resection rate. They reported an en bloc resection rate of $46 \%(6 / 13)$ and a vertical margin negative rate of $84.6 \%(11 /$ $13)$, while our en bloc resection and vertical margin negative rates were both $100 \%(10 / 10)$. However, the lesions in their study were in the $T$ stage at baseline (T1/T2/T3:6/3/2), much more advanced than the lesions in our study, and treated with EMR (mucosa/sub-mucosa: 5/5). Therefore, a simple comparison was not possible.

With regards to ESD, Saito et al. [11] reported an en bloc resection rate of $100 \%$ with a negative margin rate of $75 \%$ in four patients treated by ESD for locally recurrent esophageal cancer after CRT, indicating that the en bloc resection rate was higher than that in 17 patients treated by EMR in the same study. In their cases, they reported that it is technically difficult to treat lesions after CRT because the lesions were accompanied by fibrosis. ESD showed a higher en bloc resection rate, along with treatment outcomes of non-CRT superficial esophageal cancers, in comparison with EMR. ESD has now become the standard treatment, but difficult ESD cases exist, the important cause of which is fibrosis in the submucosa.

Usually, ESD is performed as a complete therapy for colon tumor. In this field, submucosal fibrosis produces a procedural difficulty and an accidental event might possibly occur [19, 20]. Submucosal fibrosis is thought to be a risk of technical difficulties or serious complications. Nevertheless, few histopathologi- 


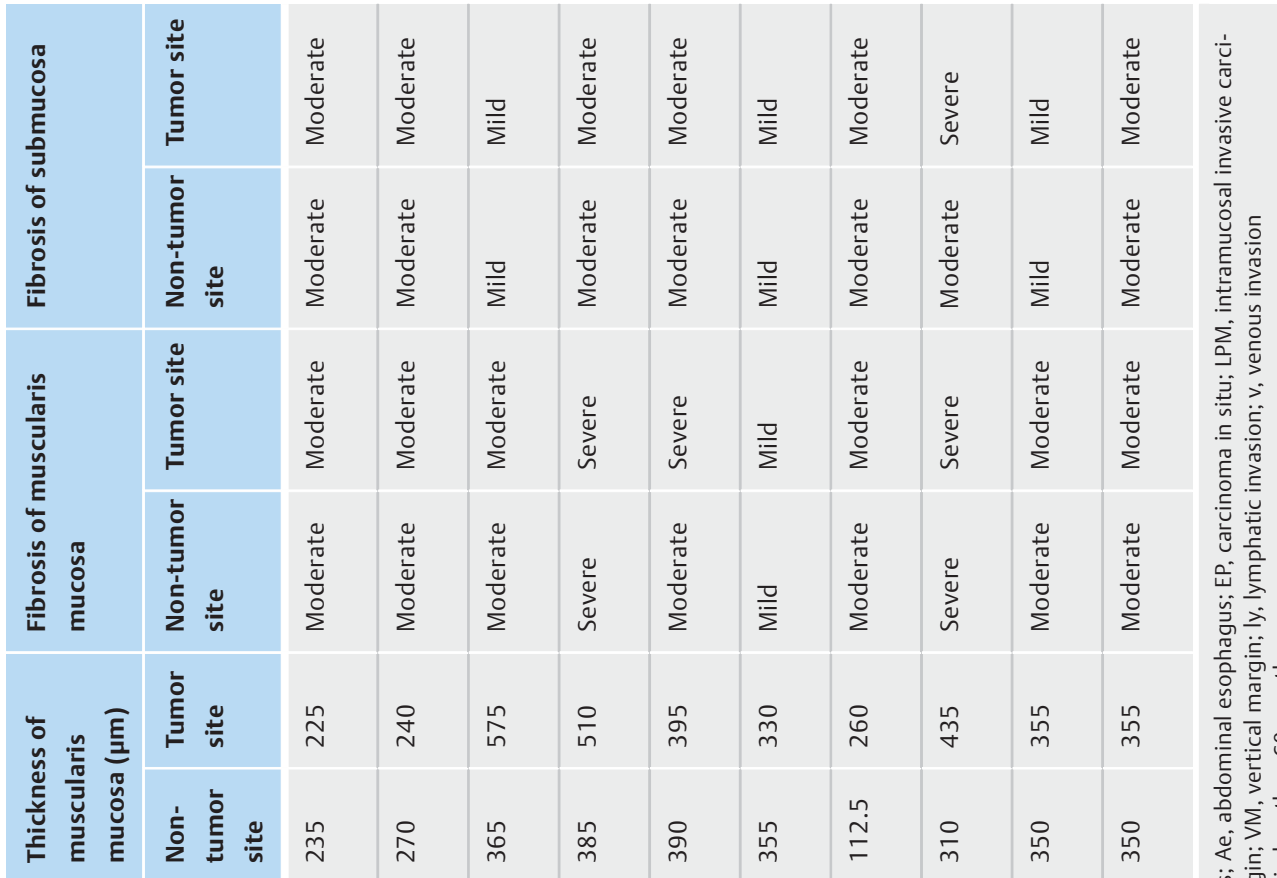

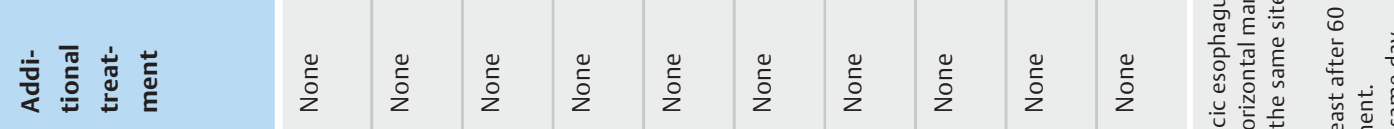

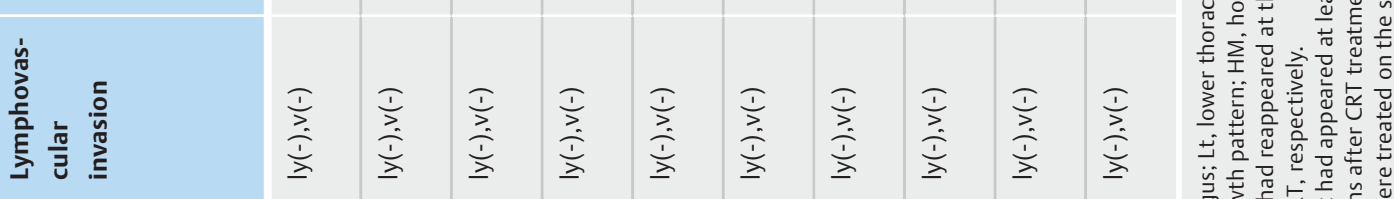

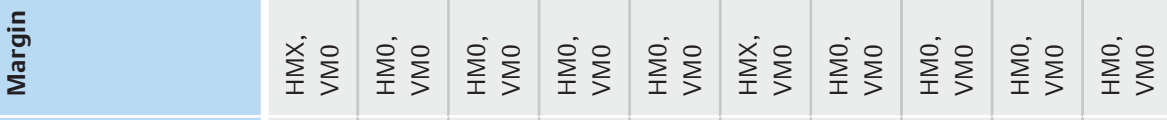

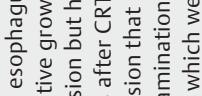

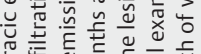

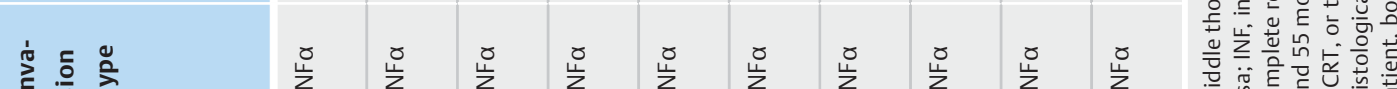

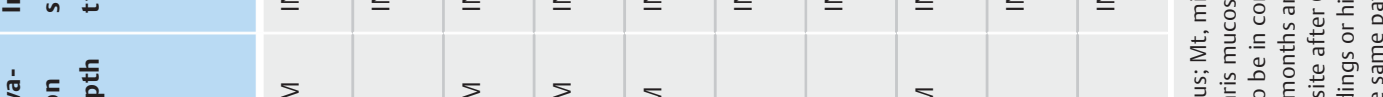

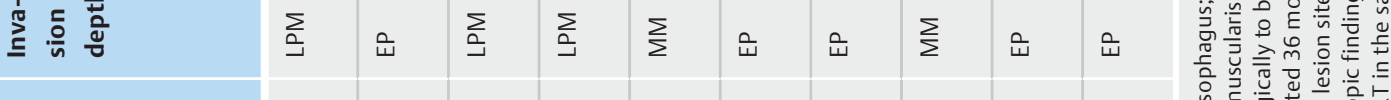

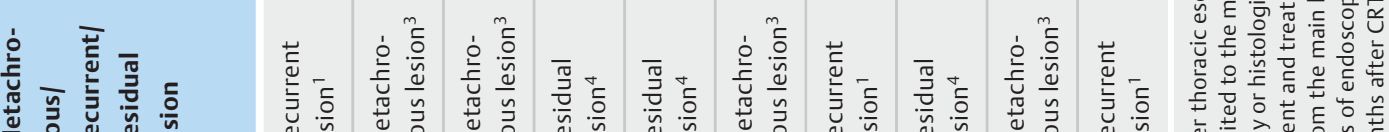

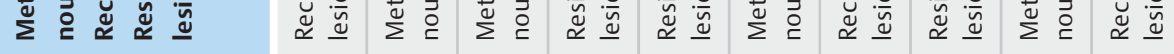

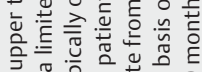

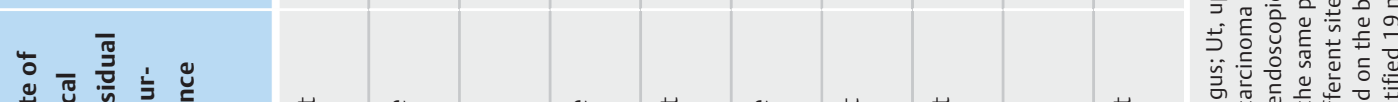

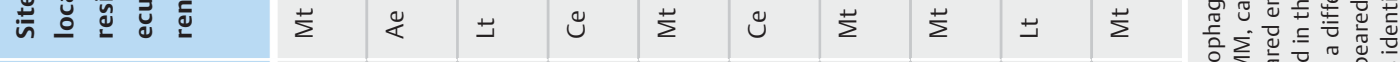

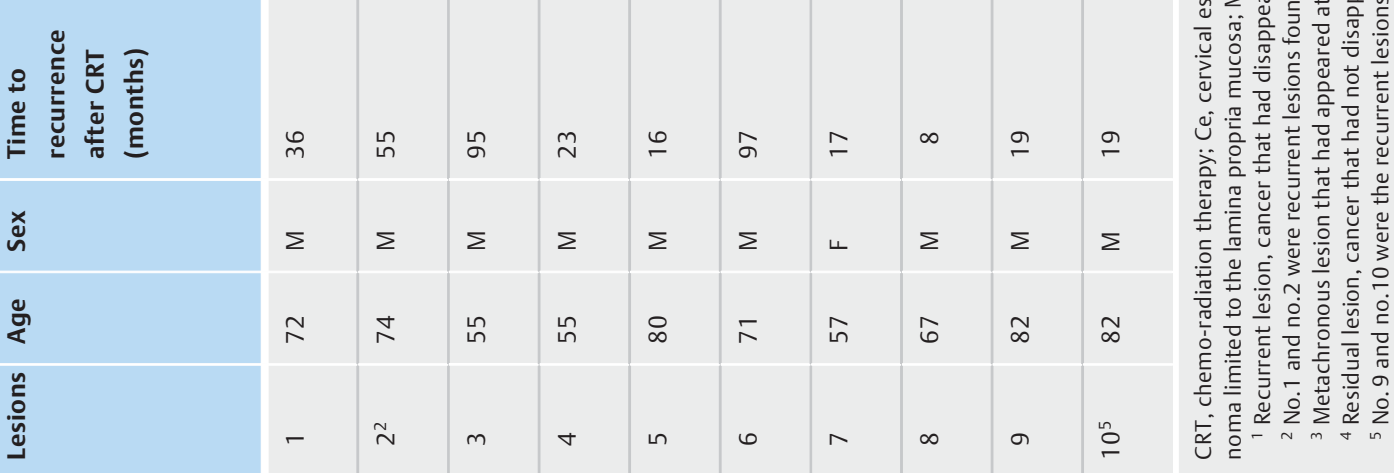


- Table 3 Comparison of clinical features of the CRT group and the non-CRT group.

\begin{tabular}{|c|c|c|c|}
\hline & CRT group & Non-CRT group & \multirow[t]{2}{*}{$P$ value } \\
\hline & $(n=10)$ & $(n=59)$ & \\
\hline Male, n (\%) & $9(90.0 \%)$ & $47(79.7 \%)$ & $P=.674^{1}$ \\
\hline Age, median(range) & $71.5(55-82)$ & $71.0(49-86)$ & $P=.831^{2}$ \\
\hline \multicolumn{4}{|l|}{ Tumor location, n (\%) } \\
\hline - CE & $2(20.0 \%)$ & $2(3.4 \%)$ & \multirow[t]{5}{*}{$P=0.70^{1}$} \\
\hline . Ut & $0(0.0 \%)$ & $8(13.6 \%)$ & \\
\hline - Mt & $4(40.0 \%)$ & $39(66.1 \%)$ & \\
\hline - Lt & $3(30.0 \%)$ & $8(13.6 \%)$ & \\
\hline - Ae & $1(10.0 \%)$ & $2(3.4 \%)$ & \\
\hline ESD procedure time(minutes), median (range) & $42(25-95)$ & $55(15-270)$ & $P=.239^{2}$ \\
\hline En bloc resection, $\mathrm{n}(\%)$ & $10(100.0 \%)$ & $59(100.0 \%)$ & - \\
\hline Complete en bloc resection, $\mathrm{n}(\%)$ & $8(80.0 \%)$ & $54(91.5 \%)$ & $P=.266^{1}$ \\
\hline Curative resection ${ }^{3}, \mathrm{n}(\%)$ & $6(60.0 \%)$ & $43(72.9 \%)$ & $P=.460^{1}$ \\
\hline Tumor length, median (range) & $550.5(195-1050)$ & $750.0(225-7252)$ & $P=.031^{2}$ \\
\hline \multicolumn{4}{|l|}{ Invasion depth, n (\%) } \\
\hline - EP & $5(50.0 \%)$ & $19(32.2 \%$ & \multirow[t]{5}{*}{$P=.555^{1}$} \\
\hline - LPM & $3(30.0 \%)$ & $29(49.2 \%)$ & \\
\hline - $\mathrm{MM}$ & $2(20.0 \%)$ & $6(10.2 \%)$ & \\
\hline - SM1 & $0(0.0 \%)$ & $2(3.4 \%)$ & \\
\hline - SM2 & $0(0.1 \%)$ & $3(5.1 \%)$ & \\
\hline \multicolumn{4}{|c|}{$\begin{array}{l}\text { CRT, chemo-radiation therapy; ESD, endoscopic submucosal dissection; Ce, cervical esophagus; Ut, upper thoracic esophagus; Mt, middle thoracic esophagus } \\
\text { Lt, lower thoracic esophagus; Ae, abdominal esophagus; EP, carcinoma in situ; LPM, intramucosal invasive carcinoma limited to the lamina propria mucosa; } \\
\text { MM, carcinoma limited to the muscularis mucosa; SM1, massive invasion ( }<200 \mu \text { into the submucosa); SM2, massive invasion ( }>200 \mu \text { into the submucosa) } \\
{ }^{1} \text { Chi-squared test } \\
{ }^{2} \text { Mann-Whitney's U test } \\
{ }^{3} \text { Curative resection means obtained complete en bloc resection and invasion depth was EP/LPM. }\end{array}$} \\
\hline
\end{tabular}

cal examinations of locally recurrent esophageal SCC after CRT have been performed.

Consequently, clarification of the pathological features of lesions after CRT and assistance in facilitating easy and safe ESD were the major objectives of the current study.

This study revealed that degree of fibrosis in the lamina propria mucosa, muscularis mucosa, and submucosa in the CRT group was higher than that in the non-CRT group in both nontumor and tumor sites and that the lamina propria mucosa and muscularis mucosa in the CRT group is thicker than that in the non-CRT group.

The acute phase of radiation therapy strongly affects the esophageal epithelium and basal cells, where cell division proceeds rapidly. In the late phase of radiation therapy, it is believed that fibroblast and inflammatory cells infiltrate into the smooth muscle, which leads to fibrosis [21]. The pathological results of this study coincide with the late-phase reaction of radiation.

Overall, the fibrotic change surrounding the lesions and thickening of the muscularis mucosae after CRT may result in procedural difficulty. In EMR, for treatment of lesions after CRT, local injection into the submucosa cannot achieve sufficient lifting of the lesions for proper snaring which results in difficulty of en bloc resection [14]. Fibrous alteration induced by radiation in the non-tumor site is a factor affecting adequate dissection of the muscularis mucosa. Conversely, ESD is suitable for treatment of lesions with fibrosis or thickening of the muscularis mucosa after CRT because dissection of lesions can be directly visualized during the procedure. However, due to thick muscular mucosae, multiple incisions were required to reach the submucosa. Ablation was widely applied including to normal and tumor areas during the incision process. As a result, tissue around the tumor was damaged ( $\triangleright$ Fig. 6 ) and histopathological analysis became impossible to perform at a horizontal margin of the two lesions (lesions 1 and 6). We thought that wide margin around the tumor, a sufficient volume of sodium hyaluronate injection into the fibrous area, and approximately deep incision would be required to complete the procedure. Use of sodium hyaluronate rather than physiological saline or glyceol solution alone is particularly important in order to ob- 
- Table4 Comparison of histopathological findings between the CRT group and non-CRT groups.

\begin{tabular}{|c|c|c|c|c|c|}
\hline & & & $\begin{array}{l}\text { CRT group } \\
(n=10)\end{array}$ & $\begin{array}{l}\text { non-CRT group } \\
(n=59)\end{array}$ & $P$ value \\
\hline \multirow{2}{*}{$\begin{array}{l}\text { Thickness of lamina propria } \\
\text { mucosa }(\mu \mathrm{m}) \text {, median (range) }\end{array}$} & Non-tumor site & & $220(130-420)$ & $180.0(30-435)$ & $P<.001^{1}$ \\
\hline & Tumor site & & $240(120-420)$ & $180.0(55-465)$ & $P<.001^{1}$ \\
\hline \multirow{4}{*}{$\begin{array}{l}\text { Fibrosis of lamina propria } \\
\text { mucosa, } \mathrm{n}(\%)\end{array}$} & \multirow[t]{2}{*}{ Non-tumor site } & None $\sim$ mild & $2(20.0 \%)$ & $43(72.9 \%)$ & \multirow[t]{2}{*}{$P=.002^{2}$} \\
\hline & & Moderate or higher & $8(80.0 \%)$ & $16(27.1 \%)$ & \\
\hline & \multirow[t]{2}{*}{ Tumor site } & None $\sim$ mild & $2(20.0 \%)$ & $42(71.2 \%)$ & \multirow[t]{2}{*}{$P=.003^{2}$} \\
\hline & & Moderate or higher & $8(80.0 \%)$ & $17(28.8 \%)$ & \\
\hline \multirow{2}{*}{$\begin{array}{l}\text { Thickness of muscularis mucosa } \\
(\mu \mathrm{m}) \text {, median (range) }\end{array}$} & Non-tumor site & & $350.0(112.5-390)$ & $150.0(50-425)$ & $P<.001^{1}$ \\
\hline & Tumor site & & $355.0(225-575)$ & $190.0(55-490)$ & $P<.001^{1}$ \\
\hline \multirow{4}{*}{$\begin{array}{l}\text { Fibrosis of muscularis mucosae, } \\
n(\%)\end{array}$} & \multirow[t]{2}{*}{ Non-tumor site } & None $\sim$ mild & $1(10.0 \%)$ & $58(98.3 \%)$ & \multirow[t]{2}{*}{$P<.001^{2}$} \\
\hline & & Moderate or higher & $9(90.0 \%)$ & $1(1.7 \%)$ & \\
\hline & \multirow[t]{2}{*}{ Tumor site } & None $\sim$ mild & $1(10.0 \%)$ & $58(98.3 \%)$ & \multirow[t]{2}{*}{$P<.001^{2}$} \\
\hline & & Moderate or higher & $9(90.0 \%)$ & $1(1.7 \%)$ & \\
\hline \multirow[t]{4}{*}{ Fibrosis of submucosa, n (\%) } & \multirow[t]{2}{*}{ Non-tumor site } & None $\sim$ mild & $3(30.0 \%)$ & $55(93.2 \%)$ & \multirow[t]{2}{*}{$P<.001^{2}$} \\
\hline & & Moderate or higfer & $7(70.0 \%)$ & $4(6.8 \%)$ & \\
\hline & \multirow[t]{2}{*}{ Tumor site } & None $\sim$ mild & $3(30.0 \%)$ & $50(84.7 \%)$ & \multirow[t]{2}{*}{$P<.001^{2}$} \\
\hline & & Moderate or higher & $7(70.0 \%)$ & $9(15.3 \%)$ & \\
\hline \multirow{3}{*}{$\begin{array}{l}\text { Diameter of submucosal vessel } \\
(\mu \mathrm{m}), \text { median (range) }\end{array}$} & \multirow[t]{2}{*}{ Artery } & Maximum vessel diameter & $510(400-630)$ & $230(60-720)$ & $P<.001^{1}$ \\
\hline & & Maximum vessel diameter & $240(170-268)$ & $122(41-500)$ & $P=.007^{1}$ \\
\hline & Vein & Maximum vessel diameter & $110(80-160)$ & $120(30-410)$ & $P=.966^{1}$ \\
\hline $\begin{array}{l}\text { CRT, chemo-radiation therapy } \\
{ }^{1} \text { Mann-Whitney's } U \text { test } \\
{ }^{2} \text { Chi-squared test }\end{array}$ & & & & & \\
\hline
\end{tabular}

tain sufficient lifting to achieve safe circumferential mucosal incision with suitable incision depth. In the CRT group, submucosal fibrosis was marked but the degree of fibrosis was moderate or less in most cases. During submucosal dissection, if the submucosa can be visually recognized, there is little difficulty with the procedure ( $\mathbf{F i g} \mathbf{6} \mathbf{d}$ ). Submucosal dissection may be more feasible using a Small-Caliber-Tip Transparent Hood [22] or the thread-traction method [23].

Radiation induces disorders such as apoptosis in vascular endothelial cells of capillary blood vessels, where cells proliferate rapidly, which causes occlusion or ischemia due to thrombosis. Vasoactive cytokines and growth factors released in the process are involved in increase of the vascular system after reperfusion [24]. In an animal experiment, due to irradiation, the diameters of arterioles and veins increased early in irradiation, shrank temporarily within a few months, and increased again over the long term [25]. This study showed that the diameter of the submucosal artery and the diameter of the lumen increased in the CRT group. In other words, in case of inadvertent damage to a blood vessel, a more severe hemorrhage may occur. A precoagulation technique [26] may become important for a prophylactic hemostasis, in case of ESD for the CRT lesions.
In this study, making an adequate circumferential mucosal incision and appropriate attention to bleeding are the only technical tips for ESD lesions after CRT. Provided that adequate level of standard techniques is applied, ESD is feasible to perform even after radiation without requiring additional advanced skill or devices. As such, all our cases were successfully resected en bloc and there was no significant difference in treatment outcomes with ESD between the CRT and non-CRT groups.

In ESD of non-CRT lesions, a higher en bloc incision rate and lower local recurrence rate than those in EMR may be attained, along with findings of a long-term follow-up period [27]. Even after post-CRT lesions, ESD may be an excellent method. In addition, in the report by Saito et al. [11], after ESD, in four cases of post-CRT lesions, the local recurrence rate was reported to 0 $\%$, despite an average follow-up period of 3 months. Takeuchi et al. [28] performed ESD in 19 cases and observed lymph node and distant metastasis in three cases over an average followup period of 54.6 months. In our examination, we also did not observe any local recurrence over the average follow-up period of 11.9 months. Takeuchi et al. [28] reported that lymph node and distant metastases occurred in cases in which massive sub- 
- Table 5 Evaluation of fibrosis.

\begin{tabular}{|c|c|c|c|c|}
\hline & & & $\begin{array}{l}\text { CRT group } \\
(n=10)\end{array}$ & $\begin{array}{l}\text { non-CRT group } \\
(n=59)\end{array}$ \\
\hline \multirow{6}{*}{ Fibrosis of lamina propria mucosa, n (\%) } & \multirow[t]{3}{*}{ Non-tumor site } & None mild & $2(20.0 \%)$ & $43(72.9 \%)$ \\
\hline & & Moderate & $3(30.0 \%)$ & $15(25.4 \%)$ \\
\hline & & Severe & $5(50.0 \%)$ & $1(1.7 \%)$ \\
\hline & \multirow[t]{3}{*}{ Tumor site } & None Mild & $2(20.0 \%)$ & $42(71.2 \%)$ \\
\hline & & Moderate & $3(30.0 \%)$ & $16(27.1 \%)$ \\
\hline & & Severe & $5(50.0 \%)$ & $1(1.7 \%)$ \\
\hline \multirow[t]{6}{*}{ Fibrosis of muscularis mucosa, n (\%) } & \multirow[t]{3}{*}{ Non-tumor site } & None Mild & $1(10.0 \%)$ & $58(98.3 \%)$ \\
\hline & & Moderate & $7(70.0 \%)$ & $1(1.7 \%)$ \\
\hline & & Severe & $2(20.0 \%)$ & $0(0.0 \%)$ \\
\hline & \multirow[t]{3}{*}{ Tumor site } & None Mild & $1(10.0 \%)$ & $58(98.3 \%)$ \\
\hline & & Moderate & $6(60.0 \%)$ & $1(1.7 \%)$ \\
\hline & & Severe & $3(30.0 \%)$ & $0(0.0 \%)$ \\
\hline \multirow[t]{6}{*}{ Fibrosis of submucosa, $\mathrm{n}(\%)$} & \multirow[t]{3}{*}{ Non-tumor site } & None Mild & $3(30.0 \%)$ & $55(93.2 \%)$ \\
\hline & & Moderate & $7(70.0 \%)$ & $4(6.8 \%)$ \\
\hline & & Severe & $0(0.0 \%)$ & $0(0.0 \%)$ \\
\hline & \multirow[t]{3}{*}{ Tumor site } & None $\sim$ Mild & $3(30.0 \%)$ & 50 (84.7\%) \\
\hline & & Moderate & $6(60.0 \%)$ & $9(15.3 \%)$ \\
\hline & & Severe & $1(10.0 \%)$ & $0(0.0 \%)$ \\
\hline
\end{tabular}

mucosal and venous invasion were observed. Accordingly, they remarked that a detailed pathological examination on the whole lesion using ESD may be useful for prediction of tumor recurrence. In the current study, safe execution of ESD was shown. With more reports on ESD in the future, the outcomes during a longer observation period and the effectiveness of ESD may be further elucidated.

Other treatment methods for post-CRT lesions besides ESD include photodynamic therapy (PDT) [29] and ablation [30], which are reported to be effective. Khangura et al. [30] conducted a meta-analysis of the endoscopic treatments for postCRT recurrence or residual lesions, including PDT, EMR, and spray cryotherapy, and reported that any of these methods may be effective for cases in which surgery is not an option. However, as in this report, the number of clinical cases was not enough. They concluded that the amount of evidence is inadequate to determine the optimal treatment.

Thus far, no comparative study has been conducted on ESD, PDT, and ablation. However, although ESD was possible up to the T1 level for resectable cases, Yano et al. reported that PDT was effective in cases up tor T2 [31]. ESD and PDT may be appropriate for recurrent or residual lesions whose depths of invasion are at the $\mathrm{T} 1$ and $\mathrm{T} 2$ levels.

Because the current study clarified that ESD may be performed safely, incision of residual lesions by ESD after CRT-in- duced tumor regression may be possible for patients with esophageal cancer. Esophageal cancer is difficult to treat using endoscopic methods alone, especially when invasion has reached the SM or deeper, or for larger lesions, especially those that are circumferential or superficial.

One of the problems with the current study is that at baseline, most recurrent or residual lesions were T1, and 4 of 10 CRT lesions were metachronous. If the tumor invasion depth at baseline is much deeper, fibrosis may become advanced, which makes ESD impossible to perform. Moreover, metachronous lesions are different histopathologically and oncologically from recurrent or residual lesions. Regarding metachronous lesions following CRT, Hamada et al. [32] reported that nine metachronous cases following CRT were successfully resected endoscopically. Murakami et al. [9] also reported the feasibility of performing ESD in metachronous esophageal lesions following CRT. In terms of performing salvage endoscopic treatment for metachronous lesions, Murakami et al. [9] emphasized the importance of detecting metachronous cancers as superficial lesions by close endoscopic observation.

However, with T2 lesions or those with deeper invasion at baseline, Takeuchi et al. [28] performed ESD on 7 such lesions among 19 cases of residual or recurrent lesions and performed en bloc resection on all the cases. Koizumi et al. [10] conducted ESD on such five cases and reported having performed en bloc 

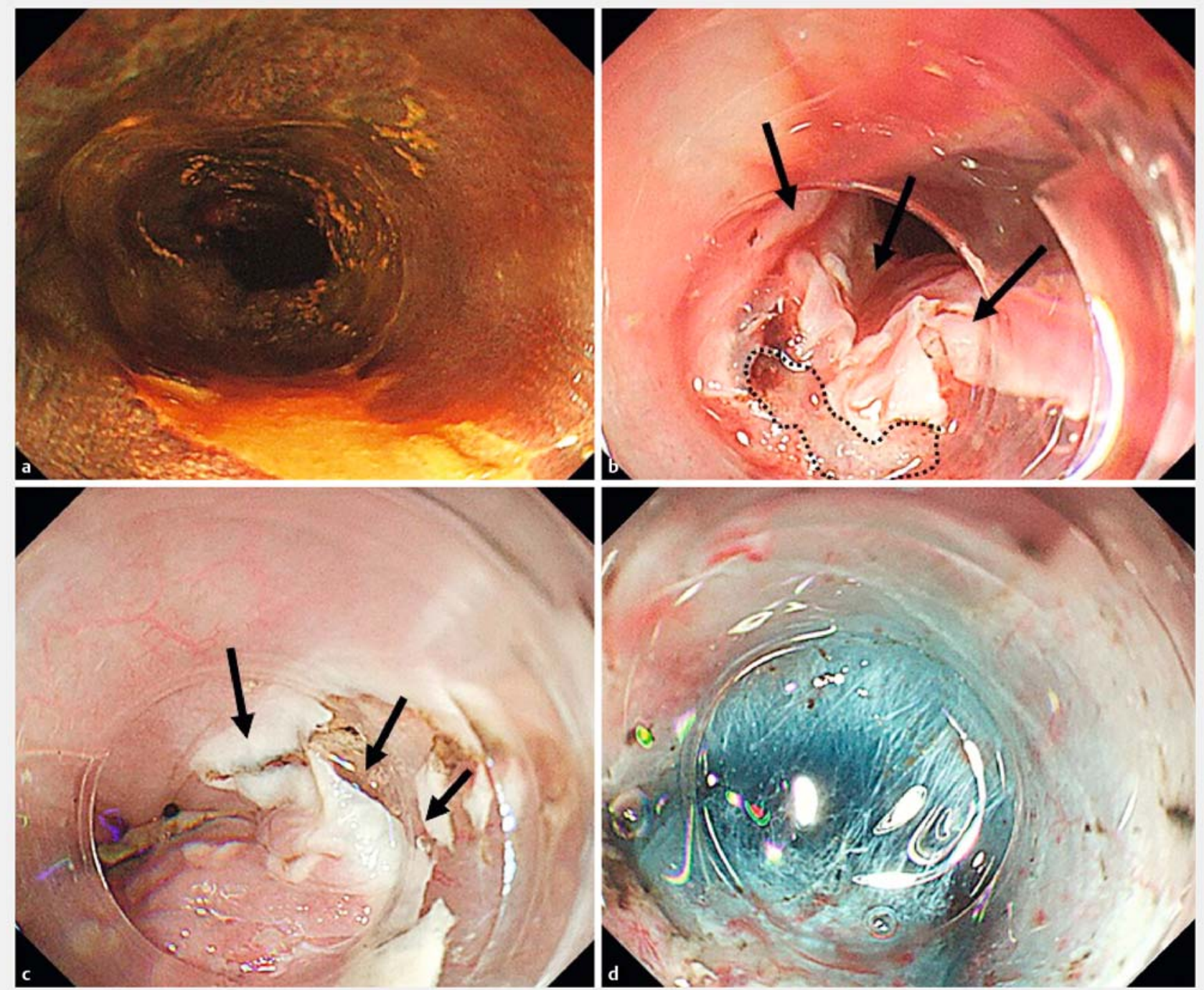

\ Fig. 6 a Recurrent lesion after CRT (Lesion 1) b Circumferential mucosal incision was incomplete due to thickened muscular mucosae ( $\ldots$ ), and tissue damage around the tumor (arrow). c Tissue damage around the tumor (arrow) was produced during the incision process. Ablation from the multiple incisions was applied including normal and tumor areas. As a result, histopathological analysis became impossible at a horizontal margin of tumor. $\mathbf{d}$ During submucosal dissection, if the submucosa can be directly visualized with adequate use of submucosal injection, the procedure itself presents little difficulty.

resection on all of them. Moreover, in our T2 cases, we found that it was not suitable for "severance" due to submucosal fibrosis but was possible for en bloc resection. Therefore, the depth of invasion of a preoperative tumor may not necessarily be related directly to the technical difficulty of ESD. In summary, in deciding whether ESD is possible, a major factor may be early detection of lesions and presence of metachronous lesions or deep invasion more than T2 may not affect the feasibility of ESD.

In the current study, submucosal fibrosis in the residual lesion was found to be more fibrotic (two of three cases were severe), and the muscularis mucosa was found to be thicker than the recurrent or metachronous lesions. Consequently, ESD of residual lesions may become slightly difficult. Even though the residual lesions are at the same $T$ stage at baseline, the tumor burden at baseline is estimated to be greater than that of recurrent or metachronous lesions, which may make fibrosis more advanced.

Although we cannot draw a firm conclusion about the clinical effectiveness or long-term results of salvage ESD due to the small case numbers in this study, we have nevertheless demonstrated the feasibility of ESD for esophageal lesions after CRT based on our pathological evaluation.

This study illustrated that ESD of lesions after CRT is not particularly challenging compared with CRT in non-CRT lesions, and no advanced skills or special devices were necessary. Therefore, ESD should be considered as one of the options for salvage therapy for esophageal cancer even after CRT.

This study does have some limitations in that it was retrospective and conducted at a single center. The number of le- 
sions was small in the CRT group. In addition, ESD was performed by two experts in ESD, which may have positively influenced treatment outcomes. No recurrent lesions of advanced stage invading submucosal or muscularis propria were included in the current study because in the majority of our patients, the stage of the original esophageal cancer was limited to T1. Therefore, further examination in patients with advanced stage disease and long-term follow-up are required.

\section{Conclusions}

Fibrosis and thickening of the muscularis mucosa needs to be recognized when ESD is performed esophageal SCC that has recurred locally after CRT. During ESD, tissue fibrosis is a risk when dissection and resection of the lesion layer are incomplete. Attention also must be paid to bleeding tendencies of arteries with increased vessel diameter. ESD for locally recurrent esophageal SCC after CRT is an effective and safe treatment when the lesion's pathological features are well understood.

\section{Acknowledgements}

The authors thank Dr. Yu Hidaka, Graduate School of Health Sciences, Okayama University, for her instruction on statistics and data analysis.

\section{Competing interests}

None

\section{References}

[1] Nakamura T, Hayashi K, Ota M et al. Salvage esophagectomy after definitive chemotherapy and radiotherapy for advanced esophageal cancer. Am J Surg 2004; 188: 261 - 266

[2] Tachimori $\mathrm{Y}$, Kanamori N, Uemura $\mathrm{N}$ et al. Salvage esophagectomy after high-dose chemotherapy for esophageal squamous cell carcinoma. J Thorac Cardiovasc Surg 2009; 137: 49-54

[3] Nomura M, Oze I, Kodaira T et al. Comparison between surgery and definitive chemotherapy for patients with resectable esophageal squamous cell carcinoma: a propensity score analysis. Int J Clin Oncol 2016; 21: $890-898$

[4] Ishihara R, Yamamoto S, lishi $\mathrm{H}$ et al. Factors predictive of tumor recurrence and survival after initial complete response of esophageal squamous cell carcinoma to definitive chemoradiotherapy. Int J Radiat Oncol Biol Phys 2010; 76: $123-129$

[5] Kato $\mathrm{H}$, Sato A, Fukuda $\mathrm{H}$ et al. A phase II trial of chemoradiotherapy for stage I esophageal squamous cell carcinoma: Japan Clinical Oncology Group Study (JCOG9708). Jpn J Clin Oncol 2009; 39: 638-643

[6] Kato K, Muro K, Minashi K et al. Phase II study of chemoradiotherapy with 5-fluorouracil and cisplatin for Stage II-III esophageal squamous cell carcinoma: JCOG trial (JCOG 9906). Int J Radiat Oncol Biol Phys 2011; 81: 684-690

[7] Stewart BW, Wild CP. World cancer report 2014. Lyon, France: IARC press; 2014

[8] Braakhuis B], Tabor MP, Kummer JA et al. A genetic explanation of Slaughter's concept of field cancerization: evidence and clinical implications. Cancer Res 2003; 63: 1727 - 1730
[9] Murakami Y, Takahashi I, Nishibuchi I et al. Long-term results of definitive concurrent chemoradiotherapy for patients with esophageal submucosal cancer (T1bNOM0). Int J Clin Oncol 2015; 20: 897 - 904

[10] Koizumi S, Jin M, Matsuhashi T et al. Salvage endoscopic submucosal dissection for the esophagus-localized recurrence of esophageal squamous cell cancer after definitive chemoradiotherapy. Gastrointest Endosc 2014; 79: 348 - 353

[11] Saito Y, Takisawa H, Suzuki H et al. Endoscopic submucosal dissection of recurrent or residual superficial esophageal cancer after chemoradiotherapy. Gastrointest Endosc 2008; 67: 355 - 359

[12] Ishii N, Suzuki K, Fujita Y. Salvage endoscopic submucosal dissection for recurrent esophageal squamous-cell carcinoma after definitive chemoradiotherapy. Clin 2011; 4: 85-88

[13] Kurokawa Y, Muto M, Minashi K et al. A phase II trial of combined treatment of endoscopic mucosal resection and chemoradiotherapy for clinical stage I esophageal carcinoma: Japan Clinical Oncology Group Study JCOG0508. Jpn J Clin Oncol 2009; 39: 686-689

[14] Yano T, Muto M, Hattori S et al. Long-term results of salvage endoscopic mucosal resection in patients with local failure after definitive chemoradiotherapy for esophageal squamous cell carcinoma. Endoscopy 2008; 40: 717-721

[15] Hatogai K, Yano T, Kojima T et al. Local efficacy and survival outcome of salvage endoscopic therapy for local recurrent lesions after definitive chemoradiotherapy for esophageal cancer. Radiat Oncol 2016; 11: 31

[16] Hwang C, Youn YH, Choi SE et al. Endoscopic Submucosal Dissection for Recurrent or Residual Superficial Esophageal Cancer after Chemoradiotherapy: Two Cases. Clin Endosc 2015; 48: 553- 557

[17] Japan Esophageal Society. Japanese classification of Esophageal Cancer. 11th Edition: part I. Esophagus 2017; 14: 1-36

[18] Makazu M, Kato K, Takisawa $\mathrm{H}$ et al. Feasibility of endoscopic mucosa resection as salvage treatment for patients with local failure after definitive chemoradiotherapy for stage IB, II, and III esophageal squamous cell cancer. Dis Esophagus 2014; 27: 42 - 49

[19] Hong SN, Byeon JS, Lee BI et al. Prediction model and risk score for perforation in patients undergoing colorectal endoscopic submucosal dissection. Gastrointest Endosc 2016; 84: 98 - 108

[20] Mizushima T, Kato M, Iwanaga I et al. Technical difficulty according to location, and risk factors for perforation, in endoscopic submucosal dissection of colorectal tumors. Surg Endosc 2015; 29: 133-139

[21] Coia LR, Myerson RJ, Tepper JE. Late effects of radiation therapy on the gastrointestinal tract. Int J Radiat Oncol Biol Phys 1995; 31 : $1213-1236$

[22] Ishii N, Horiki N, Itoh T et al. Endoscopic Submucosal Dissection With a Combination of Small-Caliber-Tip Transparent Hood and Flex Knife is a Safe and Effective Treatment for Superficial Esophageal Neoplasias. Surg. Endosc 2010; 24: 335-342

[23] Koike $\mathrm{Y}$, Hirasawa D, Fujita $\mathrm{N}$ et al. Usefulness of the thread-traction method in esophageal endoscopic submucosal dissection: randomized controlled trial. Dig Endosc 2015; 27: 303 - 309

[24] Stone HB, Coleman CN, Anscher MS et al. Effects of radiation on normal tissue: consequences and mechanisms. Lancet Oncol 2003; 4: $529-536$

[25] Goertz O, Poettgen C, Akbari A et al. New model for long-term investigations of cutaneous microcirculatory and inflammatory changes following irradiation. J Radiat Res 2015; 56: 456 - 461

[26] Tanaka S, Toyonaga T, Morita Y. Endoscopic vessel sealing: a novel endoscopic precoagulation technique for blood vessels during endoscopic submucosal dissection. Dig Endosc 2013; 25: 341 - 342

[27] Nishizawa T, Tahagi N. Endoscopic mucosal resection and endoscopic submucosal dissection: technique and new directions. Curr Opin Gastroenterol 2017; 33: 315-319 
[28] Takeuchi M, Kobayashi M, Hashimoto S. Salvage endoscopic submucosal dissection in patients with local failure after chemoradiotherapy for esophageal squamous cell carcinoma. Scand J Gastroenterol 2013; 48: $1095-1101$

[29] Kumagai K, Mariosa D, Tsai JA et al. Systematic review and meta-analysis on the significance of salvage esophagectomy for persistent or recurrent esophageal squamous cell carcinoma after definitive chemoradiotherapy. Dis Esophagus 2016; 29: $734-739$

[30] Khangura SK, Greenwald BD. Endoscopic management of esophageal cancer after definitive chemoradiotherapy. Dig Dis Sci 2013; 58: $1477-1485$
[31] Yano T, Muto M, Minashi K et al. Long-term results of salvage photodynamic therapy for patients with local failure after chemoradiotherapy for esophageal squamous cell carcinoma. Endoscopy 2011; 43: $657-663$

[32] Hamada K, Ishihara R, Yamasaki Y et al. Efficacy and Safety of Endoscopic Resection Followed by Chemoradiotherapy for Superficial Esophageal Squamous Cell Carcinoma: A Retrospective Study. Clin Transl Gastroenterol 2017; 8: 8 